\title{
Fungos conidiais na Caatinga: espécies associadas ao folhedo
}

\author{
Alisson Cardoso Rodrigues da Cruz $^{1,2}$ e Luís Fernando Pascholati Gusmão ${ }^{1}$
}

Recebido em 09/04/2008. Aceito em 30/01/2009

RESUMO - (Fungos conidiais na Caatinga: espécies associadas ao folhedo). Um inventário de fungos conidiais foi realizado em seis áreas de extrema importância biológica no bioma Caatinga. Foram coletadas 74 espécies, sendo sete novos registros para o Brasil. Heliocephala zimbabweensis Decock, V. Robert \& Masuka é reportada pela segunda vez para a ciência, Sporidesmium verrucisporum M.B. Ellis é um novo registro para o continente americano, Dicyma vesiculifera Piroz., Fusariella concinna (Syd.) S. Hughes, F. obstipa (Pollack) S. Hughes, Phaeostalagmus tenuissimus (Corda) W. Gams e Sporidesmiella claviformis P.M. Kirk são novos registros para a América do Sul. Falcocladium sphaeropedunculatum Crous \& Alfenas, Myrmecridium schulzeri var. schulzeri (Sacc.) Arzanlou, W. Gams \& Crous, Pseudodictyosporium wauense Matsush., Repetophragma inflatum (Berk. \& Ravenel) W.P. Wu são novos registros para o semi-árido brasileiro. Seis espécies foram reconhecidas como novos taxa, Brachysporiellina fecunda S.M. Leão, A.C. Cruz, R.F. Castañeda \& Gusmão, Diplococcium verruculosum A.C. Cruz, Gusmão \& R.F. Castañeda, Lobatopedis longistriatum A.C. Cruz, Gusmão, S.M. Leão-Ferreira \& R.F. Castañeda, Subramaniomyces pulcher A.C. Cruz, Gusmão \& R.F. Castañeda e foram publicadas separadamente. Os novos registros são descritos, ilustrados e comentados. Uma lista incluindo as demais espécies encontradas é apresentada.

Palavras-chave: Biodiversidade, fungos anamórficos, serapilheira, taxonomia

ABSTRACT - (Conidial fungi from Caatinga: leaf litter species). An inventory of conidial fungi was carried out in six areas of extreme biological importance within the Caatinga biome. Seventy four species were collected, with seven new records for Brazil. Heliocephala zimbabweensis Decock, V. Robert \& Masuka is recorded for the second time for the world, Sporidesmium verrucisporum M.B. Ellis is new to the American continent, Dicyma vesiculifera Piroz., Fusariella concinna (Syd.) S. Hughes, F. obstipa (Pollack) S. Hughes, Phaeostalagmus tenuissimus (Corda) W. Gams and Sporidesmiella claviformis P.M. Kirk are new to South America; Falcocladium sphaeropedunculatum Crous \& Alfenas, Myrmecridium schulzeri var. schulzeri (Sacc.) Arzanlou, W. Gams \& Crous, Pseudodictyosporium wauense Matsush. and Repetophragma inflatum are new to the Brazilian semi-arid region. Four species were recognized as new, Brachysporiellina fecunda S.M. Leão, A.C. Cruz, R.F. Castañeda \& Gusmão, Diplococcium verruculosum A.C. Cruz, Gusmão \& R.F. Castañeda, Lobatopedis longistriatum A.C. Cruz, Gusmão, S.M. Leão-Ferreira \& R.F. Castañeda, Subramaniomyces pulcher A.C. Cruz, Gusmão \& R.F. Castañeda and were published separately. The new records are described, illustrated and commented. A list including all other collected species is presented.

Key words: Anamorphic fungi, biodiversity, leaf litter, taxonomy

\section{Introdução}

O clima semi-árido submete a sua biota a condições climáticas extremas, como altas temperaturas médias anuais $\left(26-28^{\circ} \mathrm{C}\right)$ e baixas precipitações $(300-1000 \mathrm{~mm} / \mathrm{ano})$, que são irregulares e concentradas em 3 a 6 meses por ano (Prado 2003; Sampaio 1995). A vegetação dominante, denominada Caatinga, é um mosaico de florestas secas e vegetação arbustiva, com enclaves de florestas úmidas montanas e de cerrados (Tabarelli \& Silva 2003). A deciduidade das folhas é comum neste bioma e os fungos têm um importante papel na ciclagem dos nutrientes acumulados na superfície do solo, particularmente os fungos conidiais, que estão entre os principais decompositores do folhedo (Sampaio 1995; Dix \& Webster 1995).

Os estudos dos fungos conidiais que atuam na decomposição de folhas no Brasil foram iniciados por Sutton \& Hodges Jr. (1975a;b; 1976a;b;c; 1977; 1978) e Sutton (1975; 1977) que descreveram a partir do folhedo de Eucalyptus spp. novas espécies e novas ocorrências para o país. No estado de Pernambuco, Maia (1983) estudou a sucessão de fungos no folhedo de três espécies vegetais. A partir dos anos 90 alguns estudos foram realizados nos estados do Paraná e São Paulo (Grandi \& Gusmão 1998; Grandi \& Gusmão 2002a; 2002b; Grandi 1998; 1999; Gusmão et al. 2000; Gusmão 2001). No sudeste investigações resultaram na descrição de novas espécies (Castañeda-Ruiz et al. 2001; Calduch et al. 2002). Recentemente em São Paulo, Grandi \& Silva $(2003$; 2006) e Grandi (2004) relataram novas ocor- rências para o Brasil. O folhedo submerso foi investigado por Schoenlein-Crusius \& Milanez (1990) e os resultados estão sumarizados em Schoenlein-Crusius \& Grandi (2003). Schoenlein-Crusius et al. (2006) coletaram 125 espécies de fungos conidiais, sendo que oito foram reportados pela primeira vez para o Brasil. Silva (2007) coletou 33 espécies e relatou três novas ocorrências para o país.

Gusmão et al. (2006) reuniram em uma única obra informações sobre todos os fungos conidiais publicados para o semi-árido brasileiro e salientaram que o conhecimento sobre esse grupo para a região ainda é bastante restrito. Foram catalogadas 407 espécies, distribuídas em 176 gêneros. Desde então a continuidade das pesquisas resultou na descoberta de sete novas espécies para a ciência e de novos relatos de fungos conidiais para o neotrópico, para a América do Sul e Brasil (Castañeda-Ruiz et al. 2006; Marques et al. 2007; Barbosa et al. 2007; Cruz et al. 2007a; b; Gusmão et al. 2008).

A diversidade fúngica em ecossistemas áridos e semiáridos pode ser igual ou superior quando comparada à de ambientes úmidos. Isso se deve ao baixo potencial hídrico do solo, inapropriado ao crescimento de bactérias, o que torna a cadeia alimentar em sistemas áridos baseada primariamente nos fungos (Zak 1993). Para testar essa hipótese torna-se relevante o estudo de áreas no semi-árido brasileiro, especialmente envolvendo a realização de inventários micológicos.

O objetivo desse trabalho foi contribuir para expansão do conhecimento sobre os fungos conidiais associados a folhas em decomposição através do inventário em áreas de extrema

\footnotetext{
1 Universidade Estadual de Feira de Santana, Departamento de Ciências Biológicas, Laboratório de Micologia Feira de Santana, BA, Brasil

2 Autor para correspondência: alissonbio@yahoo.com.br
} 
importância biológica. Nesta publicação são descritos e ilustrados as novas ocorrências para o continente americano, América do Sul e para o semi-árido brasileiro. Além disso, é apresentada uma lista com as demais espécies encontradas.

\section{Materiais e métodos}

No período de agosto de 2006 a fevereiro 2007 foram realizadas coletas da serapilheira em cada uma das seis áreas de extrema importância biológica do bioma Caatinga (MMA 2002). As seis áreas estão localizadas nos seguintes estados, Bahia: (1) Região do Médio São Francisco (mun. Pilão Arcado); (2) Raso da Catarina (mun. Jeremoabo e Paulo Afonso), (3) Região de Senhor do Bonfim (mun. Campo Formoso e Senhor do Bonfim); Pernambuco: (4) Região de Buíque/Vale do Ipojuca (mun. Buíque); Piauí: (5) Parque Nacional Serra das Confusões (mun. Caracol); Rio Grande do Norte: (6) Região de Seridó/Borborema (mun. Serra Negra do Norte).

As amostras de folhas foram submetidas à técnica de lavagem descrita por Castañeda-Ruiz (2005). As amostras foram mantidas em câmara-úmida em temperatura ambiente e observadas diariamente em estereomicroscópio por um período de 20 dias. As estruturas reprodutivas dos fungos conidiais foram coletadas com auxílio de agulhas de ponta fina e colocadas em meio de montagem permanente com resina PVL (álcool polivinílico + lactofenol). Posteriormente as lâminas foram depositadas no Herbário da Universidade Estadual de Feira de Santana (HUEFS). A identificação foi realizada a partir de medições das estruturas de importância taxonômica e de consulta à literatura especializada. Foram incluídas descrições, comentários, distribuição geográfica e ilustrações para as novas ocorrências além de uma lista com todas as demais espécies encontradas. As ilustrações foram obtidas em câmera clara acoplada ao microscópio óptico Zeiss modelo Axioskop 40.

\section{Resultados e discussão}

Dicyma vesiculifera Piroz., Mycol. Pap. 129: 12. 1972.

Fig. 1-2

Conidióforos macronemáticos, mononemáticos, eretos, retos ou flexuosos, septados, ramificados, lisos, castanhos, 175-265 × 3-3,5 $\mu \mathrm{m}$; células em forma de bastão, hialinas, 9-16,5 × 1,5-2,5 $\mu \mathrm{m}$, vesículas apicais subglobosas, hialinas, 3,5-6 $\mu \mathrm{m}$ diâm. Ramificações retas, cilíndricas, septadas, lisas, castanho-claras, 31,5-70,5 × 1,5-2,5 $\mu \mathrm{m}$. Células conidiogênicas holoblásticas, poliblásticas, laterais, em grupos de 2-6 posicionadas em verticilos, simpodiais, cilíndricas, denticuladas, lisas, hialinas, 6-9,5 × 1-2 $\mu \mathrm{m}$. Conídios solitários, 0 -septados, elípticos, simples, secos, lisos, hialinos, $4-5 \times 1,5-2,5 \mu \mathrm{m}$.

Material examinado: BRASIL. Bahia: Pilão Arcado, $7 /$ III/2007, A.C.R. Cruz s.n. (HUEFS 130981).

Distribuição geográfica: Cuba (Castañeda-Ruiz 1986), Tanzânia (Pirozynski 1972), Nepal (Stalpers 2007).

São reconhecidas 12 espécies para o gênero (Arx 1982; Guarro \& Calvo 1983). No material examinado os conídios apresentam-se menores que os descritos por Pirozynski (1972) e Castañeda-Ruiz (1986). Dicyma vesiculifera assemelha-se a $D$. ampullifera Boulanger e D. funiculosa Guarro \& Calvo (Guaro \& Calvo 1983) pela presença de vesículas. No entanto, $D$. ampullifera é facilmente diferenciada de $D$. vesiculifera por possuir conídios verruculosos e $D$. funiculosa pela presença de vesículas menores presentes em conidióforos com ramificações restritas ao ápice. Dicyma Boulanger tem sua fase teleomórfica associada ao gênero Ascotricha Berk. (Xylariaceae, Ascomycota). Arx (1982) estudando os gêneros Basifimbria Subram. \& Lodha, Gonytrichella Emoto \& Tubaki, Hansfordia S. Hughes e Puciola de Bertoldi propôs a sinonimização destes com Dicyma. No entanto, a proposição não foi seguida por outros pesquisadores que continuam a adicionar espécies em Hansfordia, como H. catalonica Mercado, Gené \& Guarro (Gené et al. 2000). A espécie é referida pela primeira vez para a América do Sul.

Falcocladium sphaeropedunculatum Crous \& Alfenas, S. Afr. J. Bot. 63(5): 287. 1997.

Fig. 3-4

Conidióforos macronemáticos, mononemáticos ou sinematosos, eretos, retos ou flexuosos, septados, simples ou ramificados, lisos, subhialinos a hialinos, 15-90 × 2,5$3 \mu \mathrm{m}$; estipes originados na primeira ramificação ou em verticilo nas métulas, aseptados, simples, lisos, 38,5-105 × 1,5-3 $\mu \mathrm{m}$; vesículas apicais subglobosas, hialinas, 4,5-7,5 $\times 3-5 \mu \mathrm{m}$; métulas em grupos de 2-3, aseptadas, cilíndricas a cuneiformes, hialinas, 6,5-9 × 3-4,5 $\mu \mathrm{m}$. Células conidiogênicas enteroblásticas, fialídicas, terminais, em grupos de 2-5, posicionadas em verticilo na métula, determinadas, lageniformes, com colaretes curvos, lisas, hialinas, 13-17 $\times$ 2-3 $\mu \mathrm{m}$. Conídios solitários, 0 -septados, falcados, raramente com apêndice basal, simples, em mucilagem, lisos, hialinos, $12,5-15 \times 0,9-1,2 \mu \mathrm{m}$.

Material examinado: BRASIL. Bahia: Pilão Arcado, Morro do Machado, 20/II/2007, A.C.R. Cruz s.n. (HUEFS 130982).

Distribuição geográfica: Brasil (Crous et al. 1997).

O gênero apresenta quatro espécies, $F$. multivesiculatum S.F. Silveira, Alfenas, Crous \& M.J. Wingf. (Crous et al. 1994), F. sphaeropedunculatum (Crous et al. 1997), F. thailandicum Crous \& Himaman (Crous et al. 2007) e F. turbinatum Somrithipol, Sudhom, Tippawan \& E.B.G. Jones (Somrithipol et al. 2007). Os conídios do material examinado raramente apresentaram apêndice basal e são mais estreitos quando comparados à espécie-tipo (Crous et al. 1997). Falcocladium multivesiculatum e $F$. thailandicum são as espécies mais próximas de $F$. sphaeropedunculatum e podem ser diferenciadas pela forma elipsoidal da vesícula e maior comprimento do conídio, respectivamente. Crous et al. (2007) realizaram a análise filogenética de $F$. multivesiculatum, F. sphaeropedunculatum e $F$. thailandicum através da região ITS confirmando a legitimidade das três espécies, o provável polifiletismo do gênero, e o alinhamento com membros de Hypocreales (Ascomycota). Este é o primeiro relato da espécie para o semi-árido brasileiro.

Fusariella concinna (Syd.) S. Hughes, Mycol. Pap. 28: 8. 1949.

Bas.: Clasterosporium concinnum Syd., Ann. Mycol., Berl. 1933.

Fig. 5-6 
Conidióforos macronemáticos, mononemáticos, eretos, retos ou flexuosos, septados, simples ou ramificados, lisos, subhialinos, 40,5-67,5 × 2,5-4 $\mu \mathrm{m}$. Células conidiogênicas enteroblásticas, fialídicas, terminais, integradas, determinadas, cilíndricas, lisas, subhialinas. Conídios em cadeia, 3-septados, cilíndrico-fusiformes, ápices curvos, simples, secos, lisos, oliva-claros, 16,5-21 × 3-4,2 $\mu \mathrm{m}$.

Material examinado: BRASIL. Bahia: Pilão Arcado, 6/ III/2007, A.C.R. Cruz s.n. (HUEFS 130983); Pernambuco: Buíque, 17/VIII/2007, A.C.R. Cruz S.n. (HUEFS 130984).

Distribuição geográfica: Argélia (Stalpers 2007), Índia (Hughes 1949; Ellis 1971), Taiwan (Matsushima 1980) e Panamá (Goos 1997).

Segundo Hughes (1949), o gênero tem como espécietipo Fusariella atrovirens Sacc. proposta em 1884 e não Fusariella atrovirens (Berk.) Sacc. (Saccardo 1886), sendo esta última um homônimo tardio da espécie-tipo. Hughes (1949) reforça o uso de Fusariella atrovirens Sacc. devido a uma grande variação na dimensão dos conídios de Fusariella atrovirens (Berk.) Sacc.

O gênero Fusariella Sacc. possui 14 espécies (Hughes 1949; Ellis 1971; 1976; Matsushima 1985; 1993), encontradas no solo, parasitando folhas e em material vegetal em decomposição. Entre as espécies que apresentam células conidiogênicas lisas $F$. concinna é diferenciada pelos conídios oliva-claros com a presença de ápice curvo. As características do espécime estudado estão de acordo com as descrições apresentadas por Hughes (1949), Ellis (1971), Matsushima (1980) e Goos (1997). Trata-se do primeiro registro desse táxon para a América do Sul.

Fusariella obstipa (Pollack) S. Hughes, Mycol. Pap. 28: 9. 1949.

Bas.: Dendryphium obstipum Pollack, Mycologia 39: 617. 1947.

Fig. $7-8$

Conidióforos macronemáticos, mononemáticos, eretos, retos ou flexuosos, septados, simples ou ramificados, lisos, subhialinos, 40,5-100,5 × 3-4,5 $\mu \mathrm{m}$. Células conidiogênicas enteroblásticas, fialídicas, terminais, integradas, determinadas, cilíndricas, lisas, subhialinas. Conídios em cadeia, 3 -septados, curvos, cilíndrico-fusiformes, simples, secos, lisos, oliváceos, 13-18 × 4,5-7,5 $\mu \mathrm{m}$.

Material examinado: BRASIL. Bahia: Paulo Afonso, 31/I/2007, A.C.R. Cruz s.n. (HUEFS 130985); 28/I/2007, A.C.R. Cruz s.n. (HUEFS 130986).

Distribuição geográfica: Argélia (Stalpers 2007), Cuba, Índia (Matsushima 1987), Estados Unidos da América (Hughes 1949), França, Nova Caledônia, Zâmbia (Ellis 1971), Uganda (Matsushima 1981).

Fusariella atrovirens e F. bizzozeriana (Sacc.) S. Hughes assemelham-se a $F$. obstipa, contudo apresentam células conidiogênicas verruculosas e conídios com maior comprimento, respectivamente. As dimensões dos caracteres taxonômicos estão incluídas dentro dos limites da variação morfológica da espécie (Ellis 1971, Hughes 1949, Matsushima 1981, 1987). Além dessas espécies, F. atrovirens e $F$. hughesii Chab.-Frydm já foram encontradas no Brasil (Castañeda-Ruiz et al. 2003a; Cruz et al. 2007c). Fusariella obstipa é relatada pela primeira vez para a América do Sul.

Heliocephala zimbabweensis Decock, V. Robert \& Masuka, Mycologia 90: 333.

Fig. 9-10

Conidióforos macronemáticos, mononemáticos, eretos, retos, septados, simples, lisos, castanhos, 180-320 × 2-6 $\mu \mathrm{m}$. Células conidiogênicas holoblásticas, monoblásticas, terminais, evidentes, determinadas, ampuliformes, lisas, castanhoclaras, 3,5-4,5 × 1,5-2,5 $\mu \mathrm{m}$. Conídios solitários, 2-septados, elipsóide-rostrados, simples, secos, lisos, castanho-claros, $12-114 \times 3-4,5 \mu \mathrm{m}$; rostros retos 4,5-102 ×0,8-2 $\mu \mathrm{m}$, ápices podem originar células conidiogênicas secundárias.

Material examinado: BRASIL. Pernambuco: Buíque, 23/VIII/2006, A.C.R. Cruz s.n. (HUEFS 118030).

Distribuição geográfica: Zimbábue (Decock et al. 1998).

O gênero é constituído por três espécies (Kumaresan \& Srinivasan 2002). Heliocephala zimbabweensis pode ser diferenciada de $H$. natarajanii Kumaresan \& M. Sriniv. e $H$. proliferans V. Rao, K.A. Reddy \& de Hoog (Rao et al. 1984) pela presença de rostro curvo e conídio liso, respectivamente. O comprimento do conidióforo do material examinado pode alcançar dimensão superior a do conidióforo do espécimetipo (Decock et al. 1998). A espécie é referida pela segunda vez para a ciência.

Myrmecridium schulzeri var. schulzeri (Sacc.) Arzanlou, W. Gams \& Crous, Stud. Mycol. 58: 84. 2007.

Bas.: Psilobotrys schulzeri Sacc., Hedwigia 23: 126. 1884.

Sin.: Chloridium schulzerii (Sacc.) Sacc., Syll. Fung. 4: 322. 1886.

Rhinocladiella schulzeri (Sacc.) Matsush., Icon. Microfung. Mats. lect. (Kobe): 124. 1975.

Ramichloridium schulzeri var. schulzeri (Sacc.) de Hoog, Stud. Mycol. 15: 64. 1977.

Acrotheca acuta Grove, J. Bot., Lond. 54: 222. 1916.

Pleurophragmium acutum (Grove) M.B. Ellis, More Dematiaceous Hyphomycetes: 165. 1976.

Rhinotrichum multisporum Doguet, Rev. Mycol., Suppl. Colon. 17: 78. 1953 (nom. inval. Art. 36) [non Acrotheca multispora (Preuss) Sacc., Syll. Fung. 4:277. 1886].

Acladium multisporum (Doguet) Bat. \& Oliveira, Anais XIII

Congr. Soc. Bot. Brasil: 347. 1964. [1962].

Fig. 11-12

Conidióforos macronemáticos, mononemáticos, eretos, retos ou flexuosos, septados, simples, lisos, castanho nas bases, subhialino nos ápices, 165-275 × 4,5-5 $\mu \mathrm{m}$. Células conidiogênicas holoblásticas, poliblásticas, terminais e intercalares, ocasionalmente laterais, simpodiais, cilíndricas, 

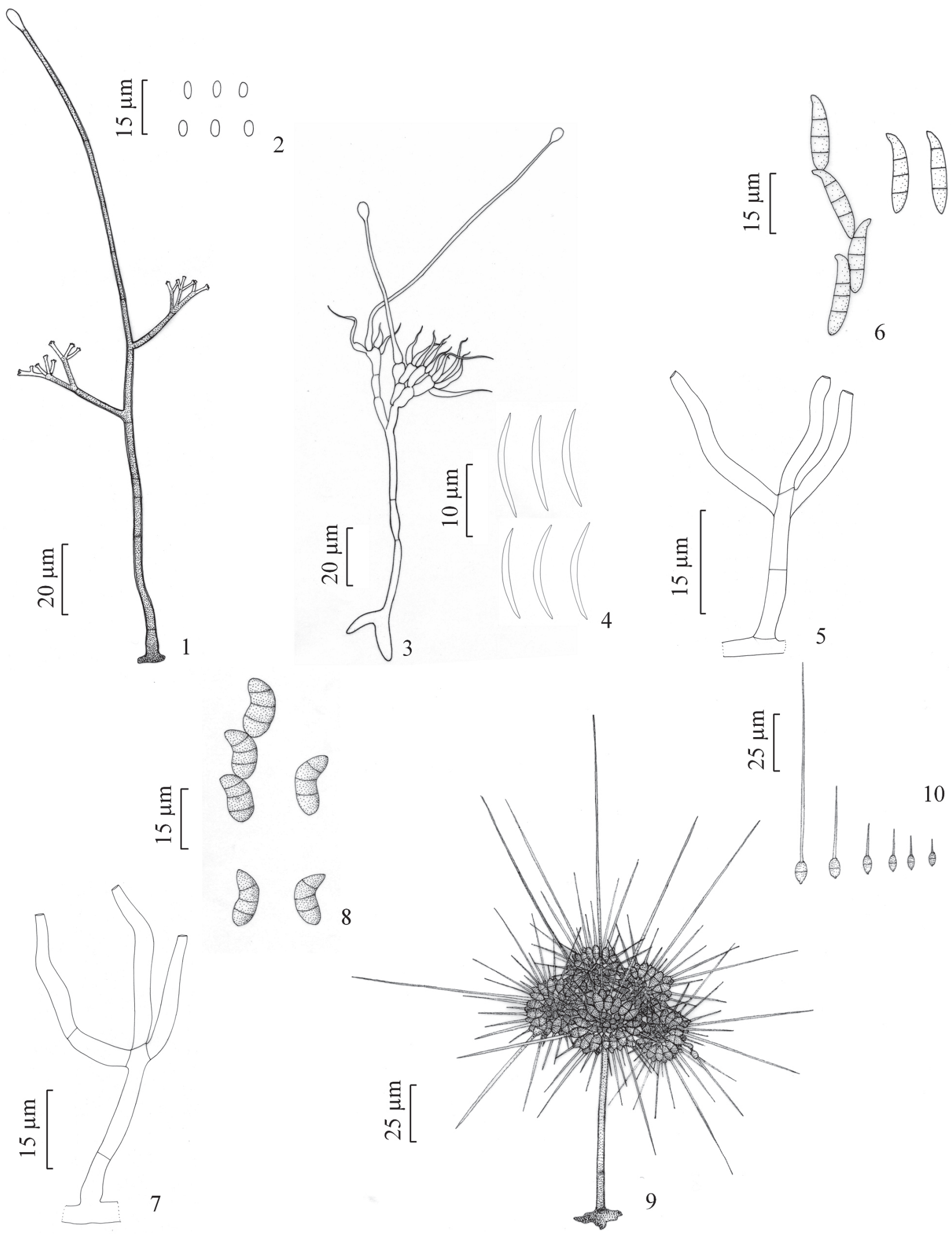

Figuras 1-10. Dicyma vesiculifera Piroz. 1. Conidióforo. 2. Conídios. 3-4. Falcocladium sphaeropedunculatum Crous \& Alfenas. 3. Conidióforo. 4. Conídios. 5-6. Fusariella concinna (Syd.) S. Hughes. 5. Conidióforo. 6. Conídios. 7-8. Fusariella obstipa (Pollack) S. Hughes. 7. Conidióforo. 8. Conídios. 9-10. Heliocephala zimbabweensis Decock, V. Robert \& Masuka. 9. Conidióforo. 10. Conídios. 
denticuladas, lisas, castanho-claras a subhialinas. Conídios solitários, 0 -septados, elipsóides a obovais, bases truncadas, simples, secos, lisos, hialinos, 5,5-14 × 3,5-5 $\mu \mathrm{m}$.

Material examinado: BRASIL. Bahia: Paulo Afonso, 2/ II/2007, A.C.R. Cruz s.n. (HUEFS 131002).

Distribuição geográfica: África do Sul, Austrália, Canadá, Holanda, Suíça, Papua-Nova Guiné, Zaire (Arzanlou et al. 2007), Antiga União das Repúblicas Socialistas Soviéticas (como Pleurothecium acutum, Farr et al. 2007), China, Estados Unidos da América (como Ramichloridium schulzeri var. schulzeri, Farr et al. 2007), Cuba (como R. schulzeri var. schulzeri, Mercado-Sierra et al. 1998), Inglaterra (como $P$. acutum, Ellis 1976), Taiwan (como R. schulzeri var. schulzeri, Matsushima 1987).

O gênero Myrmecridium Arzanlou, W. Gams \& Crous foi proposto recentemente a partir da filogenia molecular das espécies de Ramichloridium Stahel ex de Hoog (Arzanlou et al. 2007). Esse estudo sugeriu a existência de um clado formado por $R$. schulzeri e suas variedades (Incertae sedis, Sordariomycetes) e outro clado incluindo a espécietipo, R. apiculatum (J.H. Mill., Giddens \& A.A. Foster) de Hoog (Mycosphaerellaceae, Dothideomycetes). As células conidiogênicas nos espécimes brasileiros apresentaram dentículos retangulares, produzidos no ápice ou de modo intercalar, diferente dos espécimes descritos por Arzanlou et al. (2007) e Matsushima (1975) que apresentam dentículos triangulares produzidos lateralmente. Os conídios do material examinado apresentaram maior variação nas dimensões e na forma. Batista \& Oliveira (1964) descrevem Acladium multisporum no Brasil, a partir da combinação Rhinotrichum multisporum. Entretanto, essa última é considerada nome inválido (Art. 36, Greuter et al. 2003) e como conseqüência $A$. multisporum torna-se um nomem nudum. Apesar disso aceitamos a ocorrência da espécie no Brasil. Trata-se do primeiro registro desse táxon para o semi-árido brasileiro.

Phaeostalagmus tenuissimus (Corda) W. Gams, Stud. Mycol. 13: 93. 1976.

Bas.: Verticillium tenuissimum Corda, Icon. fung. (Prague) 1: 20.1837.Sin.: Verticillium apicale Berk. \& Broome, Ann. Mag. Nat. Hist., Ser. 2. 7: 101. 1851.

Verticicladium apicale (Berk. \& Broome) Sacc., Syll. fung. (Abellini) 4: 328. 1886.

Fig. 13-14

Conidióforos macronemáticos, mononemáticos, retos ou flexuosos, septados, simples, lisos, castanhos, 80-130 × 3-6 $\mu \mathrm{m}$. Células conidiogênicas enteroblásticas, fialídicas, laterais e terminais, evidentes, determinadas, lageniformes, lisas, subhialinas, 8-13 × 1,5-3 $\mu \mathrm{m}$. Conídios solitários, 0 -septados, elípticos, simples, em mucilagem, lisos, hialinos, $2-3 \times 1-1,5 \mu \mathrm{m}$.

Material examinado: BRASIL. Pernambuco: Buíque, 24/VIII/2006, A.C.R. Cruz s.n. (HUEFS 130988).
Distribuição geográfica: Canadá (como V. tenuissimum Corda, Sutton 1973), China (Tang et al. 2005), Escócia (Kirk \& Spooner 1984), Estados Unidos da América, Índia (Farr et al. 2007), Holanda, República Tcheca (Gams \& Holobová-Jechova 1976), Inglaterra (como V. tenuissimum Corda, Hughes 1951), Polônia (Chlebicki \& Chmiel 2006), Tailândia (Lumyong et al. 2003).

O gênero Phaeostalagmus W. Gams foi proposto a partir da combinação de duas espécies de Verticillium Nees, V. cyclosporus (Grove) E.W. Mason \& S. Hughes e V. tenuissimum Corda (Gams \& Holobová-Jechová 1976). Sete espécies estão incluídas em Phaeostalagmus, mas até o momento nenhuma havia sido relatada no Brasil. Phaeostalagmus tenuissimus assemelha-se a P. rossicus $\mathrm{B}$. Sutton \& Mel'nik (Sutton \& Mel'nik 1992) e a P. peregrinus Minter \& Hol.-Jech. (Minter \& Holubová-Jechová 1981) pelas células conidiogênicas em verticilos simples. $P$. rossicus apresenta células conidiogênicas maiores e conídios cilíndricos e $P$. peregrinus possui conídios maiores com base trucada. Phaeostalagmus sp. foi associado recentemente a Chaetosphaeria phaeostalacta Réblová através da cultura dos ascósporos (Reblová 2004). Este é o primeiro relato da espécie para a América do Sul.

Pseudodictyosporium wauense Matsush., in Kabayasi et al., Bull. natn. Sci. Mus., Tokyo 14(3): 473.1971

Sin.: Kamatia indica V.G. Rao \& Subhedar, Trans. Br. mycol. Soc. 66(3): 541. 1976.

Fig. 15-16

Conidióforos macronemáticos, mononemáticos, retos ou flexuosos, septados, ramificados, lisos, castanhos na base, castanho-claros no ápice, 56-106,5 × 1,5-4,5 $\mu \mathrm{m}$. Células conidiogênicas enteroblásticas, tréticas, terminais, integradas, determinadas, cilíndricas, lisas, castanho-claras. Conídios solitários, muriformes, palmados, simples, secos, lisos, castanho-claros, 16-18 × 10,5-13,5 $\mu \mathrm{m}$.

Material examinado: BRASIL. Bahia: Senhor do Bonfim, distrito Carrapichel, 21/IX/2007, A.C.R. Cruz s.n. (HUEFS 130990).

Distribuição geográfica: Brasil (Grandi \& Silva 2003), Costa do Marfim (Rambelli et al. 2004), Cuba (CastañedaRuiz 1985), Espanha, Holanda, Porto Rico, Taiwan (Stalpers 2007), Itália (Lunghini \& Quadraccia 1991), Japão (Matsushima 1975), Papua-Nova Guiné (Matsushima 1971), Peru (Matsushima 1993), Venezuela (Castañeda-Ruiz et al. 2003b).

Pseudodictyosporium Matsush. é um gênero monotípico descrito originalmente em folha em decomposição de Musa paradisiaca L. (Matasushima 1971). As características do espécime estudado estão de acordo com as descrições de Matsushima (1971, 1975) e Grandi \& Silva (2003). Tsui et al. (2006) confirmaram a sinonímia entre Kamatia indica e $P$. wauense proposta por Kirk et al. (2001) através da filogenia molecular. Tsui et al. (2006) também indicaram que a diferença entre a 
região ITS de $P$. wauense e Cheiromoniliophora elegans Tzean $\&$ J.L. Chen não seria suficiente para manter Cheiromoniliophora como um gênero distinto. Tsui et al. (2006) sugerem que Dictyosporium Corda e os gêneros mencionados pertencem à Massarinaceae (Ascomycota). A espécie foi primeiramente reportada para o Brasil por Grandi \& Silva (2003), sendo esta a primeira citação do táxon para o semi-árido brasileiro.

Repetophragma inflatum (Berk. \& Ravenel) W.P. Wu, Fung. Divers. Res. Ser. 15: 109, 2005.

Bas.: Helminthosporium inflatum Berk. \& Ravenel, Grevillea 3: 104. 1875.

Sin.: Helminthosporium subfuscum Berk. \& M.A. Curtis, Grevillea 3: 104. 1875.

Helminthosporium tiara Berk. \& Ravenel, Grevillea 3(no. 27): 104. 1875.

Helminthosporium episphaericum Cooke \& Peck, Rep. N.Y. St. Mus. nat. Hist. 29: 52. 1878.

Helminthosporium collabendum Cooke, Grevillea 17: 67. 1889.

Clasterosporium herculeum Ellis, Proc. Acad. nat. Sci. Philad.: 92. 1891.

Clasterosporium sigmoideum Ellis \& Everh., Bull. Torrey bot. Club 24: 472. 1897.

Sporidesmium inflatum (Berk. \& Ravenel) M.B. Ellis, Mycol. Pap. 70: 70. 1958.

Fig. 17-18

Conidióforos macronemáticos, mononemáticos, retos ou flexuosos, septados, simples, lisos, castanhos, ápices castanho-claros, 137,5-405 × 6-7 $\mu \mathrm{m}$. Células conidiogênicas holoblásticas, monoblásticas, terminais, integradas, com proliferações percurrentes, cilíndricas, lisas, castanho-claras. Conídios solitários, 4-5-septados, sigmóides, simples, secos, lisos, células basais trapezóides, castanho-claras, $2^{\mathrm{a}}$ e $3^{\mathrm{a}}$ células cilíndrico-trucadas, castanhas, $4^{\mathrm{a}}$ células trapezóides alongadas, castanho-claras, células apicais subuladas, subhialinas a castanho-claras, 76,5-85,5 × 13,5-16,5 $\mu \mathrm{m}$.

Material examinado: BRASIL. Piauí: Caracol, 5/ XII/2006, A.C.R. Cruz s.n. (HUEFS 130991).

Distribuição geográfica: Brasil (Grandi \& Silva 2006), China, Inglaterra (Wu \& Zhuang 2005), Cuba (MercadoSierra 1984), Estados Unidos da América, Serra Leoa (Ellis 1958), Guiné, Uganda (Ellis 1971), Índia (Farr et al. 2007), Japão (Matsushima 1975), Papua-Nova Guiné (Matsushima 1971), Peru (Matsushima 1993), Taiwan (Matsushima 1980).

O gênero Repetophragma foi proposto por Subramanian (1992) para acomodar espécies de Sporidesmium Link que apresentavam conídios eusseptados com células conidiogênicas com proliferação percurrente. Atualmente o gênero apresenta 17 espécies descritas (Wu \& Zhuang 2005; Castañeda-Ruiz et al. 2006). Sporidesmium longiphorum R.F. Castañeda \& W.B. Kendr. é a espécie que mais se assemelha a $R$. inflatum, no entanto, pode ser diferenciada pela presença de células centrais castanhas, transversalmente estriadas. $\mathrm{O}$ espécime coletado no semi-árido apresentou conidióforos maiores quando comparado aos espécimes descritos por Wu \& Zhuang (2005) e Ellis (1958). A primeira citação da espécie para o Brasil foi feita por Grandi \& Silva (2006) ainda sob o nome de Sporidesmium, portanto tratase de uma nova ocorrência para o semi-árido brasileiro. Recentemente, Castañeda-Ruiz et al. (2006) propuseram a combinação de duas espécies para o gênero, Repetophragma fasciatum (R.F. Castañeda) R.F. Castañeda, Gusmão \& Saikawa e Repetophragma filiferum (Piroz.) R.F. Castañeda, Gusmão \& Heredia, a partir de Endophragmiella fasciata (R. F. Castañeda) R. F. Castañeda e Sporidesmium filiferum Piroz., respectivamente.

Sporidesmium verrucisporum M.B. Ellis, Mycol. Pap. 70: 57. 1958.

Fig. 19-20

Conidióforos macronemáticos, mononemáticos, retos ou flexuosos, septados, simples, lisos, castanho-escuros, 46,5-165 × 3-5,5 $\mu \mathrm{m}$. Células conidiogênicas holoblásticas, monoblásticas, terminais, integradas, com proliferações percurrentes ou doliformes, cilíndricas, lisas, castanhoescuras. Conídios solitários, 6-8-septados, obclavados, retos ou levemente curvos, simples, secos, células da base castanho-escuras, verrucosos, células apicais lisas, castanho a castanho-claras, 49,5-85 $\times$ 6-10,5; base 2-3,5 $\mu \mathrm{m}$ larg.

Material examinado: BRASIL. Bahia: Jeremoabo, 29/I/2007, A.C.R. Cruz s.n. (HUEFS 130992).

Distribuição geográfica: China (Zhou et al. 2001), Nova Zelândia (Farr et al. 2007), Serra Leoa (Ellis 1958).

Sporidesmium é um gênero cosmopolita com cerca de 60 espécies (Cai et al. 2006). É caracterizado pelos conídios euseptados produzidos em células conidiogênicas sem proliferação ou apresentando proliferação lageniforme ou doliforme (Wu \& Zhuang 2005). Apesar dos conídios mais estreitos e 6-8-septados, as características apresentadas pelo espécime estudado enquadram-se em $S$. verrucisporum (Ellis 1958). Sporidesmium flagellatum (S. Hughes) M.B. Ellis e S. tropicale M.B. Ellis apresentam conídios verruculosos, podendo ser diferenciados pela presença de rostro e pelas maiores dimensões, respectivamente. Shenoy et al. (2006) analisaram filogeneticamente a subunidade maior do DNA ribossômico e a segunda subunidade maior da RNA polimerase II e forneceram fortes evidências que os gêneros que compõe atualmente Sporidesmium "sensu lato" são parafiléticos e estão posicionados entre as duas maiores classes de Ascomycota, Dothideomycetes e Sordariomycetes. A espécie é referida pela primeira vez para a América.

Sporidesmiella claviformis P.M. Kirk, Trans. Br. Mycol. Soc. 79(3): 479. 1982.

Fig. 21-22

Conidióforos macronemáticos, mononemáticos, retos ou flexuosos, septados, simples, lisos, castanho-escuros, ápices 


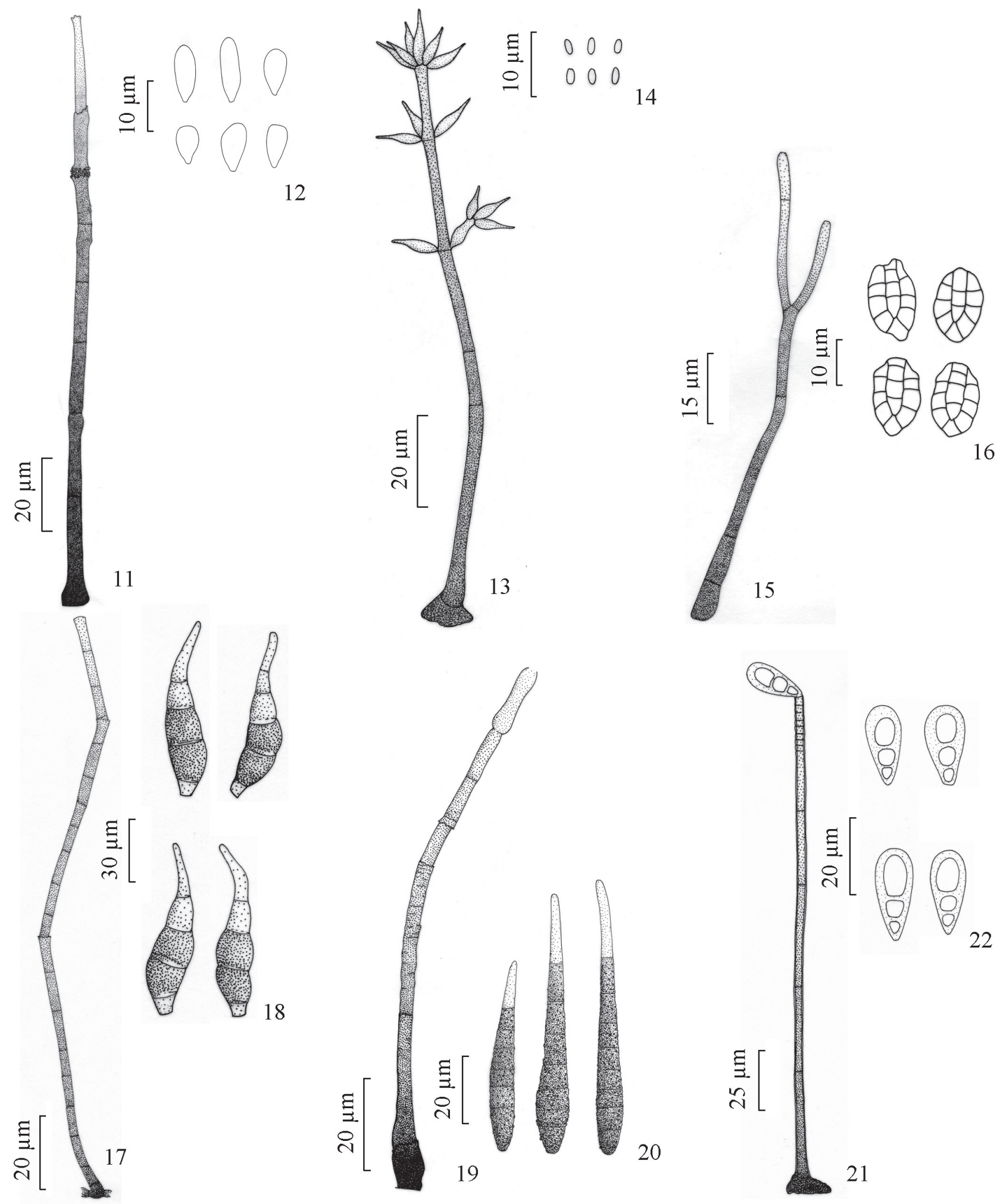

Figuras 11-22. 11-12. Myrmecridium schulzeri var. schulzeri (Sacc.) Arzanlou, W. Gams e Crous. 11. Conidióforo. 12. Conídios. 13-14. Phaeostalagmus tenuissimus (Corda) W. Gams. 13. Conidióforo. 14. Conídios. 15-16. Pseudodictyosporium wauense Matsush 15. Conidióforo. 16. Conídios. 17-18. Repetophragma inflatum (Berk. \& Ravenel) W.P. Wu. 17. Conidióforo. 18. Conídios. 19-20. Sporidesmium verrucisporum M.B. Ellis. 19. Conidióforo. 20. Conídios 21-22. Sporidesmiella claviformis P.M. Kirk. 21. Conidióforo. 22. Conídios. 
castanho-claros, 162-249 × 4,5-5,5 $\mu \mathrm{m}$. Células conidiogênicas holoblásticas, monoblásticas, terminais, integradas, com proliferações percurrentes anelídicas, cilíndricas, lisas, castanhas. Conídios solitários, 2-pseudoseptados, obovais, bases truncadas ou afiladas, simples, secos, subhialinos, 20,5-24 × 9-10,5 $\mu \mathrm{m}$; bases 0,5-4 larg. $\mu \mathrm{m}$.

Material examinado: BRASIL. Pernambuco: Buíque, 28/VIII/2006, A.C.R. Cruz s.n. (HUEFS 130993).

Distribuição geográfica: China (Wu \& Zhuang 2005), Cuba (Castañeda-Ruiz \& Kendrick 1990), Malásia (Kuthubutheen \& Nawawi 1993), Reino Unido (Kirk 1982).

Sporidesmiella claviformis é a espécie-tipo do gênero Sporidesmiella P.M. Kirk, que apresenta 24 espécies e duas variedades (Wu \& Zhuang 2005, Yanna et al. 2001). O material examinado apresentou conídios com maiores dimensões do que os referidos por Wu \& Zhuang (2005), Kuthubutheen \& Nawawi (1993) e Kirk (1982). A base do conídio afilada é uma novidade para a espécie. Sporidesmiella caribensis R.F. Castañeda pode ser diferenciada de $S$. claviformis por possuir conídios mais largos e célula externa castanha (CastañedaRuiz 1988). Três espécies deste gênero foram relatadas no Brasil: S. hyalosperma var. hyalosperma (Corda) P.M. Kirk (Grandi 1990), Sporidesmiella parva (M.B. Ellis) P.M. Kirk (Barbosa et al. 2007) e Sporidesmiella vignalensis W.B. Kendr. \& R.F. Castañeda (Marques et al. 2007). Esta é a primeira citação do táxon para a América do Sul.

\section{Lista com as demais espécies encontradas}

Acrogenospora sphaerocephala (Berk. \& Broome) M.B. Ellis, Dematiaceous Hyphomycetes (Kew): 114. 1971.

Material examinado: BRASIL. Bahia: Jeremoabo, Mata das Pororocas, sobre folha em decomposição de Palmae, 30/I/2007, A.C.R. Cruz s.n. (HUEFS 129304).

Alternaria alternata (Fr.) Keissl., Beih. Bot. Zbl., Abt. 1 29(2): 434. 1912.

Material examinado: BRASIL. Piauí: Caracol, 28/ XI/2006, A.C.R. Cruz s.n. (HUEFS 129289); Rio Grande do Norte: Serra Negra do Norte, 26/X/2006, A.C.R. Cruz s.n. (HUEFS 129290).

Atrosetaphiale flagelliformis Matsush., Matsush. Mycol. Mem. 8: 14. 1995.

Material examinado: BRASIL. Bahia: Jeremoabo, Mata das Pororocas, 30/I/2007, A.C.R. Cruz S.n. (HUEFS 129291); Pilão Arcado, Barra do Iu, 27/II/2007, A.C.R. Cruz s.n. (HUEFS 129292); Piauí: Caracol, 07/XII/2006, A.C.R. Cruz s.n. (HUEFS 129293).

Beltrania rhombica Penz., Michelia 2(no. 8): 474. 1882.

Material examinado: BRASIL. Bahia: Campo Formoso, Mata da Esterzinha, 05/X/2006, A.C.R. Cruz s.n. (HUEFS 129297); Paulo Afonso, 16/I/2007, A.C.R. Cruz s.n. (HUEFS 129294); Paulo Afonso, 28/I/2007, A.C.R. Cruz
S.n. (HUEFS 129295); Pilão Arcado, Quirino, 19/II/2007, A.C.R. Cruz s.n. (HUEFS 129296); Pernambuco: Buíque, 23/VIII/2006, A.C.R. Cruz s.n. (HUEFS 129298); Buíque, sobre folha em decomposição 05/IX/2006, A.C.R. Cruz s.n. (HUEFS 129299); Piauí: Caracol, 24/XI/2006, A.C.R. Cruz s.n. (HUEFS 129300); Caracol, 27/XI/2006, A.C.R. Cruz s.n. (HUEFS 129301); Caracol, 06/XII/2006, A.C.R. Cruz s.n. (HUEFS 129302); Rio Grande do Norte: Serra Negra do Norte, 25/X/2006, A.C.R. Cruz s.n. (HUEFS 129303).

Beltraniella portoricensis (F. Stevens) Piroz. \& S. D. Patil, Can. J. Bot. 48:575. 1970.

Material examinado: BRASIL. Bahia: Jeremoabo, Mata das Pororocas, 30/I/2007, A.C.R. Cruz s.n. (HUEFS 129305); Pernambuco: Buíque, 23/VIII/2006, A.C.R. Cruz s.n. (HUEFS 129306).

Beltraniopsis ramosa R.F. Castañeda, Revta Jardín bot. Nac., Univ. Habana 6(1): 53. 1985.

Material examinado: BRASIL. Bahia: Senhor do Bonfim, 28/IX/2006, A.C.R. Cruz s.n. (HUEFS 129308).

Bipolaris sorokiniana (Sacc.) Shoemaker, Can. J. Bot. 37(5): 884. 1959.

Material examinado: BRASIL. Piauí: Caracol, 24/ XI/2006, A.C.R. Cruz s.n. (HUEFS 129330).

Brachysporiellina fecunda S.M. Leão, A.C. Cruz, R.F. Castañeda \& Gusmão, Mycotaxon 104: 310. 2008.

Material examinado: BRASIL. Bahia: Senhor do Bonfim, 10/X/2006, S.M. Leão-Ferreira s.n. (HUEFS 120866).

Chaetopsina fulva Rambelli, Diagn. IV 3: 5. 1956.

Material examinado: BRASIL. Bahia: Jeremoabo, Mata das Pororocas, 18/I/2007, A.C.R. Cruz S.n. (HUEFS 129311); Senhor do Bonfim, Serra da Maravilha, 19/IX/2006, A.C.R. Cruz s.n. (HUEFS 129312).

Circinotrichum papakurae S. Hughes \& Piroz., N.Z. J1 Bot. 9(1): 40. 1971.

Material examinado: BRASIL. Bahia: Senhor do Bonfim, 19/X/2006, A.C.R. Cruz s.n. (HUEFS 129316).

Curvularia eragrostidis (Henn.) J.A. Mey., Publ. Inst. nat. Étude agron. Congo belge, Sér. sci. 75: 183. 1959.

Material examinado: BRASIL. Piauí: Caracol, 21/XI/2006, A.C.R. Cruz s.n. (HUEFS 129321); Caracol, 23/XI/2006, A.C.R. Cruz s.n. (HUEFS 129322); Rio Grande do Norte: Serra Negra do Norte, 26/X/2006, A.C.R. Cruz s.n. (HUEFS 129323).

Curvularia lunata (Wakker) Boedijn, Bull. Jard. Bot. Buitenz, 3 Sér. 13(1): 127. 1933.

Material examinado: BRASIL. Rio Grande do Norte: Serra Negra do Norte, 13/XI/2006, A.C.R. Cruz s.n. (HUEFS 129324); Serra Negra do Norte, 14/XI/2006, A.C.R. Cruz s.n. (HUEFS 129325). 
Dictyochaeta britannica (M.B. Ellis) Whitton, McKenzie \& K.D. Hyde, Fungal Diversity 4: 137. 2000.

Material examinado: BRASIL. Bahia: Paulo Afonso, 31/I/2007, A.C.R. Cruz s.n. (HUEFS 129376).

Dictyochaeta novae-guineensis (Matsush.) A.I. Romero, Bol. Soc. Argent. Bot. 22: 76. 1983.

Material examinado: BRASIL. Bahia: Senhor do Bonfim, fazenda Passaginha, 5/X/2006, A.C.R. Cruz s.n. (HUEFS 129366); Senhor do Bonfim, fazenda Passaginha, 18/X/2006, A.C.R. Cruz s.n. (HUEFS 129367); Pernambuco: Buíque, 31/VIII/2006, A.C.R. Cruz s.n. (HUEFS 129368), Buíque, 8/IX/2006, A.C.R. Cruz s.n. (HUEFS 129369).

Dictyochaeta pluriguttulata Kuthub. \& Nawawi, Mycol. Res. 95: 1212. 1991.

Material examinado: BRASIL. Pernambuco: Buíque, 18/VIII/2006, A.C.R. Cruz s.n. (HUEFS 129372).

Dictyochaeta simplex (S. Hughes \& W.B. Kendr.) Hol.Jech., Folia geobot. phytotax. 19: 434. 1984.

Material examinado: BRASIL. Pernambuco: Buíque, 28/VIII/2006, A.C.R. Cruz s.n. (HUEFS 129363), Buíque, 8/IX/2006, A.C.R. Cruz s.n. (HUEFS 129364).

Dictyochaeta triseptata (Matsush.) R.F. Castañeda, Fungi Cubenses: 8. 1986.

Material examinado: BRASIL. Bahia: Jeremoabo, Mata das Pororocas, A.C.R. Cruz S.n. (HUEFS 129374).

Dicyma pulvinata (Berk. \& M.A. Curtis) Arx, Gen. Fungi Sporul. Cult., Edn 3 (Vaduz): 316. 1981.

Material examinado: BRASIL. Bahia: Pilão Arcado, sobre folha em decomposição de Mauritia flexuosa L., 22/ II/2007, A.C.R. Cruz s.n. (HUEFS 129327).

Diplococcium verruculosum A.C. Cruz, Gusmão \& R.F. Castañeda, Mycotaxon 102: 34. 2007.

Material examinado: BRASIL. Bahia: Senhor do Bonfim, 11/X/2006, A.C.R. Cruz s.n. (HUEFS 120872).

Dischloridium laeense (Matsush.) B. Sutton, Kavaka 4: 47. 1977.

Material examinado: BRASIL. Bahia: Jeremoabo, Mata das Pororocas, 18/I/2007, A.C.R. Cruz s.n. (HUEFS 129328).

Drechslera halodes (Drechsler) Subram. \& B.L. Jain, Curr. Sci. 35: 354. 1966.

Material examinado: BRASIL. Rio Grande do Norte: Serra Negra do Norte, 26/XI/2006, A.C.R. Cruz s.n. (HUEFS 129329).

Ellisembia brachypus (Ellis \& Everh.) Subram., Proc. Indian Nat. Sci. Acad., Part B. Biol. Sci. 58: 183. 1992.

Material examinado: BRASIL. Pernambuco: Buíque, 25/VIII/2006, A.C.R. Cruz s.n. (HUEFS 129334).
Epicoccum nigrum Link, Magazin Ges. naturf. Freunde, Berlin 7: 32. 1815.

Material examinado: BRASIL. Bahia: Pilão Arcado, 18/ II/2007, A.C.R. Cruz s.n. (HUEFS 129331).

Gonytrichum chlamydosporium var. chlamydosporium G.L. Barron \& G.C. Bhatt, Mycopath. Mycol.Appl. 32: 126. 1967. Material examinado: BRASIL. Pernambuco: Buíque, 17/VIII/2006, A.C.R. Cruz s.n. (HUEFS 129335); Buíque, sobre folha em decomposição 18/VIII/2006, A.C.R. Cruz s.n. (HUEFS 129336); Buíque, sobre folha em decomposição 21/ VIII/2006, A.C.R. Cruz s.n. (HUEFS 129337).

Gonytrichum macrocladum (Sacc.) S. Hughes, Trans. Br. mycol. Soc. 34: 565. 1951.

Material examinado: BRASIL. Pernambuco: Buíque, 23/VIII/2006, A.C.R. Cruz s.n. (HUEFS 129339); Buíque, 26/VIII/2006, A.C.R. Cruz s.n. (HUEFS 129340).

Gyrothrix microsperma (Höhn.) Piroz., Mycol. Pap. 84: 14. 1962.

Material examinado: BRASIL. Bahia: Jeremoabo, 29/I/2007, A.C.R. Cruz s.n. (HUEFS 129341); Pilão Arcado, Quirino, 2/III/2007, A.C.R. Cruz s.n. (HUEFS 129343); Pernambuco: Buíque, 31/VIII/2006, A.C.R. Cruz s.n. (HUEFS 129345).

Helicosporium griseum Berk. \& M.A. Curtis, Grevillea 3: 51. 1874.

Material examinado: BRASIL. Pernambuco: Buíque, 21/VIII/2006, A.C.R. Cruz s.n. (HUEFS 129353).

Helicosporium virescens (Pers.) Sivan., Bitunicate Ascomycetes and their Anamorphs: 591-592. 1984.

Material examinado: BRASIL. Pernambuco: Buíque, 11/X/2006, A.C.R. Cruz s.n. (HUEFS 129359).

Idriella lunata P.E. Nelson \& S. Wilh., Mycologia 48(4): 550. 1956.

Material examinado: BRASIL. Piauí: Caracol, 9/ XII/2006, A.C.R. Cruz s.n. (HUEFS 129378).

Idriella ramosa Matsush., Bull. Natn. Sc. Mus., Tokio 14(3):466. 1971.

Material examinado: BRASIL. Bahia: Senhor do Bonfim, Serra da Maravilha, 25/IX/2006, A.C.R. Cruz s.n. (HUEFS 129382).

Idriella setiformis R.F. Castañeda \& G.R.W. Arnold. Revta. Jardín Bot. Nac., Univ. Habana 6: 50. 1985.

Material examinado: BRASIL. Bahia: Paulo Afonso, 31/I/2007, A.C.R. Cruz s.n. (HUEFS 129379); Senhor do Bonfim, 19/X/2006, A.C.R. Cruz s.n. (HUEFS 129380); Senhor do Bonfim, fazenda Passaginha, 18/X/2006, A.C.R. Cruz s.n. (HUEFS 129381). 
Janetia mangiferae S. Hughes \& Cavalc., Can. J. Bot. 61(8): 2224. 1983.

Material examinado: Brasil. Piauí: Caracol, 23/XI/2007, A.C.R. Cruz s.n. (HUEFS 129394).

Kionochaeta ramifera (Matsush.) P.M. Kirk \& B. Sutton, Trans. Br. mycol. Soc. 85(4): 715. 1986.

Material examinado: BRASIL. Bahia: Senhor do Bonfim, Serra da Maravilha, 11/X/2006, A.C.R. Cruz s.n. (HUEFS 129383).

Lobatopedis longistriatum A.C. Cruz, Gusmão, S.M. LeãoFerreira \& R.F. Castañeda, Mycotaxon 102: 37. 2007.

Material examinado: BRASIL. Pernambuco: Buíque, 16/VIII/2006, A.C.R. Cruz s.n. (HUEFS 120870).

Memnoniella echinata (Rivolta) Galloway, Trans. Br. mycol. Soc. 18(2): 165 (1933)

Material examinado: BRASIL. Bahia: Paulo Afonso, 30/I/2007, A.C.R. Cruz s.n. (HUEFS 129384); Pilão Arcado, 8/III/2007, A.C.R. Cruz s.n. (HUEFS 129385); Piauí: Caracol, 6/XII/2007, A.C.R. Cruz s.n. (HUEFS 129387); Rio Grande do Norte: Serra Negra do Norte, 14/XI/2006, A.C.R. Cruz s.n. (HUEFS 129388).

Myrothecium gramineum Lib., Pl. Crypt., Arduennae: 380. 1837.

Material examinado: BRASIL. Bahia: Paulo Afonso, 28/I/2007, A.C.R. Cruz s.n. (HUEFS 129399); Paulo Afonso, 1/II/2007, A.C.R. Cruz s.n. (HUEFS 129400).

Myrothecium leucotrichum (Peck) M.C. Tulloch, Mycol. Pap. 130: 12. 1972.

Material examinado: BRASIL. Bahia: Paulo Afonso, 31/I/2007, A.C.R. Cruz s.n. (HUEFS 129395); Pilão Arcado, 6/III/2007, A.C.R. Cruz s.n. (HUEFS 129396); Pernambuco: Buíque, 26/VIII/2006, A.C.R. Cruz s.n. (HUEFS 129397); Piauí: Caracol, 5/XII/2006, A.C.R. Cruz s.n. (HUEFS 129398).

Myrothecium roridum Tode ex Fr., Fung. mecklenb. sel. (Lüneburg) 1: 25. 1790.

Material examinado: BRASIL. Pernambuco: Buíque, 23/VIII/2006, A.C.R. Cruz s.n. (HUEFS 129401); Piauí: Caracol, 1/XII/2006, A.C.R. Cruz s.n. (HUEFS 129402); Caracol, 15/XII/2006, A.C.R. Cruz s.n. (HUEFS 129403); Rio Grande do Norte: Serra Negra do Norte, 14/XI/2006, A.C.R. Cruz s.n. (HUEFS 129404).

Myrothecium verrucaria (Alb. \& Schwein.) Ditmar ex Fr.: 7 (1813) [1817].

Material examinado: BRASIL. Bahia: Pilão Arcado, Barra do Iu, 26/II/2007, A.C.R. Cruz s.n. (HUEFS 129406); Piauí: Caracol, 14/XII/2006, A.C.R. Cruz s.n. (HUEFS 129407); Caracol, 7/XII/2006, A.C.R. Cruz s.n. (HUEFS 129408).
Neojohnstonia minima Gusmão \& Grandi, Mycotaxon 80: 98. 2001.

Material examinado: BRASIL. Bahia: Jeremoabo, 26/I/2007, A.C.R. Cruz s.n. (HUEFS 129409).

Nigrospora sphaerica (Sacc.) E.W. Mason, Trans. Br. mycol. Soc. 12: 158. 1927.

Material examinado: BRASIL. Piauí: Caracol, 27/ XI/2006, A.C.R. Cruz s.n. (HUEFS 129410); Caracol, 4/ XII/2006, A.C.R. Cruz s.n. (HUEFS 129411); Caracol, 12/ XII/2006, A.C.R. Cruz s.n. (HUEFS 129412).

Periconia cookei E.W. Mason \& M.B. Ellis, Mycol. Pap. 56: 72.1953.

Material examinado: BRASIL. Bahia: Paulo Afonso, 2/ II/2007, A.C.R. Cruz s.n. (HUEFS 129414); Senhor do Bonfim, 19/X/2006, A.C.R. Cruz s.n. (HUEFS 129415); Pernambuco: Buíque, 8/IX/2006, A.C.R. Cruz s.n. (HUEFS 129416); Piauí: Caracol, 27/XI/2006, A.C.R. Cruz s.n. (HUEFS 129417); Caracol, 11/XII/2006, A.C.R. Cruz s.n. (HUEFS 129419).

Phaeoisaria clematidis (Fuckel) S. Hughes, Can. J. Bot. 36: 794.1958.

Material examinado: BRASIL. Pernambuco: Buíque, 26/VIII/2006, A.C.R. Cruz s.n. (HUEFS 129420).

Pithomyces chartarum (Berk. \& M.A. Curtis) M.B. Ellis, Mycol. Pap. 76: 13. 1960.

Material examinado: BRASIL. Bahia: Paulo Afonso, 31/I/2007, A.C.R. Cruz s.n. (HUEFS 129421); Paulo Afonso, 28/I/2007, A.C.R. Cruz S.n. (HUEFS 129423); Pilão Arcado, Quirino, 21/II/2007, A.C.R. Cruz s.n. (HUEFS 129424); Piauí: Caracol, 4/XII/2006, A.C.R. Cruz s.n. (HUEFS 129425); Caracol, 1/XII/2006, A.C.R. Cruz s.n. (HUEFS 129426).

Repetophragma filiferum (Piroz.) R.F. Castañeda, Gusmão \& Heredia, Mycotaxon 95:269. 2006.

Material examinado: BRASIL. Pernambuco: Buíque, 23/VIII/2006, A.C.R. Cruz s.n. (HUEFS 129431); Buíque, 1/IX/2006, A.C.R. Cruz s.n. (HUEFS 129432).

Spegazzinia tessarthra (Berk. \& M.A. Curtis) Sacc., Syll. Fung. (Abellini) 4: 758. 1886.

Material examinado: BRASIL. Pernambuco: Buíque, 8/ IX/2006, A.C.R. Cruz s.n. (HUEFS 129433); Piauí: Caracol, 4/XII/2006, A.C.R. Cruz s.n. (HUEFS 129434); Caracol, 6/ XII/2006, A.C.R. Cruz s.n. (HUEFS 129435); Caracol, 14/ XII/2006, A.C.R. Cruz s.n. (HUEFS 129436).

Speiropsis scopiformis Kuthub. \& Nawawi, Trans. Br. mycol. Soc. 89(4): 584. 1987.

Material examinado: BRASIL. Bahia: Campo Formoso, Mata da Esterzinha, 5/X/2006, A.C.R. Cruz s.n. (HUEFS 129437); Senhor do Bonfim, Distrito de Carrapichel, 9/X/2006, A.C.R. Cruz s.n. (HUEFS 129439). 
Sporidesmiella hyalosperma var. hyalosperma (Corda) P.M. Kirk, Trans. Br. mycol. Soc. 79(3): 481. 1982.

Material examinado: BRASIL. Pernambuco: Buíque, 21/VIII/2006, A.C.R. Cruz s.n. (HUEFS 129443).

Stachybotrys nephrospora Hansf., Proc. Linn. Soc. London 155: 45.1943 [1942-43].

Material examinado: BRASIL. Bahia: Paulo Afonso, 28/I/2007, A.C.R. Cruz s.n.(HUEFS 129443); Pilão Arcado, morro do Machado, 7/III/2007, A.C.R. Cruz s.n. (HUEFS 129444); Senhor do Bonfim, Distrito de Carrapichel, 10/X/2006, A.C.R. Cruz s.n. (HUEFS 129445); Senhor do Bonfim, Serra da Maravilha, 10/X/2006, A.C.R. Cruz s.n. (HUEFS 129446); Pernambuco: Buíque, 24/VIII/2006, A.C.R. Cruz s.n. (HUEFS 129447); Piauí: Caracol, 28/ XI/2006, A.C.R. Cruz s.n. (HUEFS 129448); Rio Grande do Norte: Serra Negra do Norte, 26/X/2006, A.C.R. Cruz s.n. (HUEFS 129449); Serra Negra do Norte, 27/X/2006, A.C.R. Cruz s.n. (HUEFS 129450).

Stachybotrys globosa P.C. Misra \& S.K. Srivast., Trans. Br. mycol. Soc. 78(3): 556. 1982.

Material examinado: BRASIL. Bahia: Pilão Arcado, 7/ III/2007, A.C.R. Cruz s.n. (HUEFS 125380);

Stachybotrys parvispora S. Hughes, Mycol. Pap. 48: 74. 1952.

Material examinado: BRASIL. Piauí: Caracol, 9/ XII/2006, A.C.R. Cruz S.n. (HUEFS 129461).

Subramaniomyces pulcher A.C. Cruz, Gusmão \& R.F. Castañeda, Mycotaxon 102: 26. 2007.

Material examinado: BRASIL. Pernambuco: Buíque, 25/VIII/2006, A.C.R. Cruz s.n. (HUEFS 120863).

Tetraploa aristata Berk. \& Broome, Ann. Mag. Nat. Hist., Ser. 2 5: 459. 1850.

Material examinado: BRASIL. Bahia: Paulo Afonso, $2 /$ II/2007, A.C.R. Cruz s.n. (HUEFS 129462).

Thozetella cristata Piroz. \& Hodges, Can. J. Bot. 51(1): 168. 1973.

Material examinado: BRASIL. Bahia: Campo Formoso, 5/X/2006, A.C.R. Cruz s.n. (HUEFS 129463); Pilão Arcado, Morro do Machado, 7/III/2007, A.C.R. Cruz s.n. (HUEFS 129464); Pernambuco: Buíque, 17/VIII/2006, A.C.R. Cruz s.n. (HUEFS 129465); Buíque, 21/VIII/2006, A.C.R. Cruz s.n. (HUEFS 129466); Piauí: Caracol, 11/XII/2006, A.C.R. Cruz s.n. (HUEFS 129467).

Thozetella cubensis R.F. Castañeda \& G.R.W. Arnold, Revta. Jardín bot. Nac., Univ. Habana 6(1): 51. 1985.

Material examinado: BRASIL. Bahia: Pilão Arcado, 21/II/2007, A.C.R. Cruz s.n. (HUEFS 129468); Senhor do Bonfim, fazenda Passaginha, 4/X/2006, A.C.R. Cruz s.n.
(HUEFS 129469); Senhor do Bonfim, 18/X/2006, A.C.R. Cruz s.n. (HUEFS 129470).

Torula herbarum (Pers.) Link, Magazin Ges. naturf. Freunde, Berlin 3(1-2): 19. 1809.

Material examinado: BRASIL. Bahia: Paulo Afonso, 2/ II/2007, A.C.R. Cruz s.n. (HUEFS 129471); Pernambuco: Buíque, 21/VIII/2006, A.C.R. Cruz s.n. (HUEFS 129473); Buíque, 6/IX/2006, A.C.R. Cruz s.n. (HUEFS 129474); Buíque, 8/IX/2006, A.C.R. Cruz s.n. (HUEFS 129475).

Uberispora heteroseptata R.F. Castañeda, Guarro \& Cano, Mycotaxon 59: 461. 1996.

Material examinado: BRASIL. Pernambuco: Buíque, 28/VIII/2006, A.C.R. Cruz s.n. (HUEFS 125356).

Vermiculariopsiella immersa (Desm.) Bender, Mycologia 24: 412. 1932.

Material examinado: BRASIL. Bahia: Jeremoabo, 30/I/2007, A.C.R. Cruz s.n. (HUEFS 129476); Paulo Afonso, 16/I/2007, A.C.R. Cruz s.n. (HUEFS 129477).

Verticicladium trifidum Preuss, Linnaea 24. 1851.

Material examinado: BRASIL. Bahia: Pilão Arcado, Morro do Machado, 20/II/2007, A.C.R. Cruz s.n. (HUEFS 129479); Piauí: Caracol, 1/XII/2007, A.C.R. Cruz s.n. (HUEFS 129480).

Wiesneriomyces laurinus (Tassi) P.M. Kirk, Trans. Br. mycol. Soc. 82(4):7248. 1984.

Material examinado: BRASIL. Bahia: Pilão Arcado, Quirino, 2/III/2007, A.C.R. Cruz s.n. (HUEFS 129481); Pilão Arcado, Morro do Machado, 7/III/2007, A.C.R. Cruz s.n. (HUEFS 129482); Senhor do Bonfim, Distrito de Carrapichel, 9/X/2006, A.C.R. Cruz s.n. (HUEFS 129483); Senhor do Bonfim, 20/X/2006, A.C.R. Cruz s.n. (HUEFS 129484); Pernambuco: Buíque, 8/XI/2006, A.C.R. Cruz s.n. (HUEFS 129485); Piauí: Caracol, 11/XII/2006, A.C.R. Cruz s.n. (HUEFS 129486).

Zygosporium gibbum (Sacc., M. Rousseau \& E. Bommer) S. Hughes, Can. J. Bot. 36: 825. 1958.

Material examinado: BRASIL. Piauí: Caracol, 5/ XII/2006, A.C.R. Cruz s.n. (HUEFS 130975).

Zygosporium masonii S. Hughes, Mycol. Pap. 44: 15. 1951. Material examinado: BRASIL. Bahia: Pilão Arcado, Brejinho, 1/III/2007, A.C.R. Cruz s.n. (HUEFS 129487); Pilão Arcado, Morro do Machado, 21/III/2007, A.C.R. Cruz s.n. (HUEFS 129488).

\section{Agradecimentos}

Os autores agradecem ao Programa de Pesquisa em Biodiversidade do Semi-árido pelo suporte financeiro (PPBio semi-árido/Ministério da Ciência e Tecnologia) e aos Drs. Rafael Castañeda-Ruiz, David W. Minter, Gregorio 
Delgado, Josep Guarro, Kevin Hyde, Acelino Alfenas, Uwe Braun, Carlos Inácio, Sayanh Somrithipol, Eric Mckenzie, Antonio Hernández-Gutiérrez, as Dras. Marizeth Groenewald, Rosely Piccolo Grandi, Barbara Paulus, Astrid Ferrer e a Msc. Priscila Silva, pela generosidade no envio de literatura. O primeiro autor agradece ao Conselho Nacional de Desenvolvimento Científico e Tecnológico (CNPq) pela bolsa de mestrado concedida, à Fundação de Amparo à Pesquisa do Estado da Bahia pelo apoio financeiro (Proc. APR071/2007) e ao Programa de Pós-graduação em Botânica (PPGBot/UEFS).

\section{Referências bibliográficas}

Arx, J.A. von. 1982. The genus Dicyma, its synonyms and related fungi. Proceedings van de Koninklijke Nederlandse Akademie van Wetenschappen 85: 21-28.

Arzanlou, M.; Groenewald, J.Z.; Gams, W.; Braun, U.; Shin, H.-D \& Crous, P.W. 2007. Phylogenetic and morphotaxonomic revision of Ramichloridium and allied genera. Studies in Mycology 58: 57-93.

Barbosa, F.R.; Gusmão, L.F.P.; Castañeda-Ruiz, R.F.; Marques, M.F.O. \& Maia, L.C. 2007. Conidial fungi from the semi-arid Caatinga biome of Brazil. New species Deightoniella rugosa \& Diplocladiella cornitumida with new records for the neotropics. Mycotaxon 102: 39-49.

Batista, A.C. \& Oliveira, S. de 1964. Dois fungos de interêsse para a alergologia. Pp: 346-350. In: Anais do XIII Congresso da Sociedade Botânica do Brasil. Recife. 1962. Sociedade Botânica do Brasil.

Cai, L.; Hyde, K.D \& Tsui, C.K.M. 2006. Genera of Freshwater Fungi. Hong Kong, Fungal Diversity Press.

Calduch, M.; Gene, J.; Stchigel, A.M. \& Guarro, J. 2002. New species of Dictyochaetopsis and Paraceratocladium from Brazil. Mycologia 94: 1071-1077.

Castañeda-Ruiz, R.F. 1985. Deuteromycotina de Cuba. Hyphomycetes III. Havana, Instituto de Investigaciones Fundamentales em Agricultura Tropical "Alejandro de Humboldt".

Castañeda-Ruiz, R.F. 1986. Fungi Cubenses. Havana, Instituto de Investigaciones Fundamentales em Agricultura Tropical "Alejandro de Humboldt".

Castañeda-Ruiz, R.F. 1988. Fungi Cubenses III. Havana, Instituto de Investigaciones Fundamentales em Agricultura Tropical "Alejandro de Humboldt".

Castañeda-Ruiz, R.F. 2005. Metodología en el estudio de los hongos anamorfos. Pp. 182-183. In: Anais do V Congresso Latino Americano de Micologia. Brasília.

Castañeda-Ruiz, R.F., Gené, J. \& Guarro, J. 2001. A new species of Rhexoampullifera from leaf litter from Brazil. Mycologia 93: 168-170.

Castañeda-Ruiz, R.F.; Guarro, J.; Velázquez-Noa, S. \& Gené, J. 2003a. A new species of Minimelanolocus and some hyphomycete records from rain forests in Brazil. Mycotaxon 85: 231-239.

Castañeda-Ruiz, R.F.; Iturriaga, T.; Minter, D.W.; Saikawa, M., Vidal, G. \& Velázquez-Noa, S. 2003b. Microfungi from Venezuela, a new species of Brachydesmiella, a new combination, and new records. Mycotaxon 85: 211-229.

Castañeda-Ruiz, R.F.; Gusmão, L.F.P.; Heredia-Abarca, G. \& Saikawa, M. 2006. Some hyphomycetes from Brazil. Two new species of Brachydesmiella, two new combinations for Repetophragma, and new records. Mycotaxon 95: 261-270.

Castañeda-Ruiz, R.F. \& Kendrick, B. 1990. Conidial Fungi from Cuba: II. University of Waterloo Biology series 33: 1-61.

Chlebicki, A. \& Chmiel, M.A. 2006. Microfungi of Carpinus betulus from Poland I. Annotated list of microfungi. Acta Mycologica 41: 253-278.

Crous, P.W.; Groenewald, J.Z. \& Himaman, W. 2007. Falcocladium thailandicum. Fungal Planet 18.

Crous, P.W.; Kendrick, W.B. \& Alfenas, A.C. 1997. New species of hyphomycetes associated with Eucalyptus. South African Journal of Botany 63: 286-290.

Crous, P.W.; Wingfield, M.J.; Alfenas, A.C. \& Silveira, S.F. 1994. Cylindrocladium naviculatum sp. nov., and two new vesiculate hyphomycete genera, Falcocladium and Vesicladiella. Mycotaxon 50: $441-458$.

Cruz, A.C.R.; Gusmão, L.F.P. \& Castañeda-Ruiz, R.F. 2007a. Conidial fungi from the semi-arid Caatinga biome of Brazil. Subramaniomyces pulcher sp. nov. and notes on Sporidesmium circinophorum. Mycotaxon 102: 25-32.

Cruz, A.C.R.; Gusmão, L.F.P.; Leão-Ferreira, S.M. \& Castañeda-Ruiz, R.F. 2007b. Conidial fungi from the semi-arid Caatinga biome of Brazil. Diplococcium verruculosum sp. nov. and Lobatopedis longistriatum sp. nov. Mycotaxon 102: 33-38.

Cruz, A.C.R.; Marques, M.F.O. \& Gusmão, L.F.P. 2007c. Fungos anamórficos (Hyphomycetes) da Chapada Diamantina: novos registros para a Bahia e o Brasil. Acta Botanica Brasilica 21: 847-855.

Decock, C.; Robert, V. \& Masuka, A. 1998. Heliocephala zimbabweensis sp. nov. from southern Africa. Mycologia 90: 330-333.

Dix, N.J. \& Webster, J. 1995. Fungal Ecology. London, Chapman \& Hall.

Ellis, M.B. 1958. Clasterosporium and some allied DematiaceaePhragmosporae. I. Mycological Paper 70: 1-89.

Ellis, M.B. 1971. Dematiaceous Hyphomycetes. Commonwealth Mycological Institute, Kew. England.

Ellis, M.B. 1976. More Dematiaceous Hyphomycetes. Commonwealth Mycological Institute. Kew. England.

Farr, D.F.; Rossman, A.Y.; Palm, M.E. \& McCray, E.B. 2007. Fungal Databases, Systematic Botany \& Mycology Laboratory, ARS, USDA. Disponível em: http://nt.arsgrin.gov/fungaldatabases/. (Acesso em 14/ novembro/2007).

Gams, W. \& Holobová-Jechová, V. 1976. Chloridium and some other dematiaceous hyphomycetes growing on decaying wood. Studies in Mycology 13: 1-97.

Gené, J.; Mercado-Sierra, A. \& Guarro, J. 2000. Dactylaria cazorlii and Hansfordia catalonica, two new hyphomycetes from litter in Spain. Mycological Research 104: 1404-1407.

Goos, R.D. 1997. Fungi of Barro Colorado Island, adjacent Panama, and the Cali region of Colombia. Mycotaxon 64: 375-383.

Grandi, R.A.P. 1990. Hyphomycetes decompositores 1. Espécies associadas às raízes de Calathea stromata (Horticultural). Revista Brasileira de Biologia 50: 123-132.

Grandi, R.A.P. 1998. Hyphomycetes decompositores do folhedo de Alchornea triplinervia (Spreng.) Müll. Arg. Hoehnea 25: 133-148.

Grandi, R.A.P. 1999. Hifomicetos decompositores do folhedo de Euterpe edulis Mart. Hoehnea 26: 87-101.

Grandi, R.A.P. 2004. Anamorfos da serapilheira nos Vales dos Rios Moji e Pilões, município de Cubatão, São Paulo, Brasil. Hoehnea 31: $225-238$.

Grandi, R.A.P. \& Gusmão, L.F.P. 1998. A técnica da lavagem sucessiva de substratos de plantas como subsídio para estudos da associação fungo/substrato e diversidade de Hyphomycetes nos ecossistemas. IV Simpósio de Ecossistemas Brasileiros. ACIESP 104: 80-90.

Grandi, R.A.P. \& Gusmão, L.F.P. 2002a. Hyphomycetes decompositores do folhedo de Tibouchina pulchra Cogn. Revista Brasileira de Botânica 25: 79-87.

Grandi, R.A.P. \& Gusmão, L.F.P. 2002b. O gênero Subulispora (Fungos Mitospóricos-Hyphomycetes) sobre folhas em decomposição no estado de São Paulo, Brasil. Hoehnea 29: 31-36.

Grandi, R.A.P. \& Silva, T.V. 2003. Hyphomycetes sobre folhas em decomposição de Caesalpinia echinata Lam.: ocorrências novas para o Brasil. Revista Brasileira de Botânica 26: 489-493.

Grandi, R.A.P. \& Silva, T.V. 2006. Fungos Anamorfos decompositores do folhedo de Caesalpinia echinata Lam. Revista Brasileira de Botânica 29: 275-287.

Greuter, W.; McNeil, J.; Barrie, F.R.; Burdet, H.M.; Demoulin, V.; Filgueiras, T.S.; Nikolson, D.H.; Silva, P.C.; Skog, J.E.; Trehane, P.; Turland, N.J.; Hawksworth, D.L. 2003. Código Internacional de Nomenclatura Botânica (Código de Saint Louis). São Paulo, Instituto de Botânica.

Guarro, J. \& Calvo, M.A. 1983. Dicyma funiculosa sp. nov. from Spain. Nova Hedwigia 37: 641-649.

Gusmão, L.F.P. 2001. Espécies de Cylindrocladium (Fungi-Hyphomycetes) associadas a folhas de Miconia cabussu Hoehne. Sitientibus. Série Ciências Biológicas 1: 120-125.

Gusmão, L.F.P.; Barbosa, F.R. \& Barbosa, F.F. 2006. Fungos Conidiais. Pp: 27-47. In: L.F.P. Gusmão; L.C. Maia (Org.). Diversidade e caracterização dos fungos no semi-árido. Recife, Associação Plantas do Nordeste. 
Gusmão, L.F.P.; Leão-Ferreira S.M.; Marques M.F.O. \& Almeida D.A.C. 2008. New species and records of Paliphora from the Brazilian semiarid region. Mycologia 100: 292-295.

Gusmão, L.F.P.; Grandi, R.A.P. \& Milanez, A. I. 2000. A new species of Beltraniopsis from Brazil, with a key to the known species. Mycological Research 104: 251-253.

Hughes, S.J. 1949. Studies on Micro-Fungi. I. The genus Fusariella Saccardo. Mycological Papers 28: 1-11.

Hughes, S.J. 1951. Studies in Micro-Fungi. XI. Some Hyphomycetes which produce phialides. Mycological Papers 45: 1-36.

Kirk, P.M. 1982. New or interesting microfungi VI. Sporidesmiella gen. nov. (Hyphomycetes). Transactions of the British Mycological Society 79: 479-489.

Kirk, P.M.; Cannon, P.F.; David, J.C. \& Stalpers, J.A. 2001. Ainsworth and Bisby's Dictionary of the fungi. Wallingford, CABI.

Kirk, P.M. \& Spooner, B.M. 1984. An account of the fungi of Arran, Gigha and Kintyre. Kew Bulletin 38: 503-597.

Kumaresan, V. \& Srinivasan, M. 2002. Heliocephala natarajanii sp. nov. from Índia. Cryptogamie Mycologie 23: 329-333.

Kuthubutheen, A.J. \& Nawawi, A. 1993. Three new and several interesting species of Sporidesmiella from submerged litter in Malaysia. Mycological Research 97: 1305-1314.

Lumyong, P.; Photita, W.; McKenzie, E.H.C.; Hyde, K.D. \& Lumyong, S. 2003. Saprobic fungi on dead wild banana. Mycotaxon 85: 345-346.

Lunghini, D. \& Quadraccia, L. 1991. Contributo alla conoscenza degli Ifomiceti del Parco Nazionale d'Abruzzo. Giornale Botanico Italiano 125: 797-815.

Maia, L.C. 1983. Sucessão de fungos em folhedo de floresta tropical úmida. Recife, Editora Universitária, Universidade Federal de Pernambuco.

Marques, M.F.O.; Barbosa, F.R.; Gusmão, L.F.P.; Castañeda-Ruiz, R.F. \& Maia, L.C. 2007. Conidial fungi from the semi-arid Caatinga biome of Brazil. Cubasina microspora sp. nov., a note on C. albo-fusca, and some new records for South America. Mycotaxon 102: 17-23.

Matsushima, T. 1971. Microfungi of the Solomon Islands and PapuaNew Guinea. Kobe, Published by the author.

Matsushima, T. 1975. Icones Microfungorum a Matsushima Lectorum. Kobe, Published by the author.

Matsushima, T. 1980. Matsushima Mycological Memoirs n. 1. Kobe, Published by the author.

Matsushima, T. 1981. Matsushima Mycological Memoirs n. 2. Kobe, Published by the author

Matsushima, T. 1985. Matsushima Mycological Memoirs n. 4. Kobe, Published by the author.

Matsushima, T. 1987. Matsushima Mycological Memoirs n. 5. Kobe, Published by the author.

Matsushima, T. 1993. Matsushima Mycological Memoirs n. 7. Kobe, Published by the author.

Mercado-Sierra, A. 1984. Hifomicetes Demaciáceos de Sierra del Rosario, Cuba. Havana, Editorial Academica.

Mercado-Sierra, A.; Gené, J.; Figueras, M.J.; Rodrígues, K. \& Guarro, J. 1998. New or rare Hyphomycetes from Cuba. IX. Some species from Pinar del Río Province. Mycotaxon 68: 417-426.

Minter, D.W. \& Holubová-Jechová, V. 1981. New or interesting Hyphomycetes on decaying pine litter from Czechoslovakia. Folia Geobotanica et Phytotaxonomica, Praha 16: 195-217.

MMA (Mistério do Meio Ambiente). 2002. Avaliação e identificação de áreas e ações prioritárias para a conservação, utilização sustentável e repartição dos benefícios da biodiversidade nos biomas brasileiros. Brasília, MMA/SBF

Pirozynski, K.A. 1972. Microfungi of Tanzania. I. Miscellaneous fungi on oil palm. II. New Hyphomycetes. Mycological Papers 129: 1-65.

Prado, D.E. 2003. As caatingas da América do Sul. Pp. 3-73. In: I.R. Leal, M. Tabarelli \& J.M.C. Silva (eds.) Ecologia e conservação da Caatinga. Recife, Editora Universitária, Universidade Federal de Pernambuco.

Rambelli, A.; Mulas, B. \& Pasqualetti, M. 2004. Comparative studies on microfungi in tropical ecosystems in Ivory Coast forest litter: behaviour on different substrata. Mycological Research 108: 325-336.

Rao, V.; Reddy, K.A. \& De Hoog, G.S. 1984. Heliocephala, a new genus of dematiaceous Hyphomycetes. Persoonia 12: 239-242.
Reblová, M. 2004. Four new species of Chaetosphaeria from New Zealand and redescription of Dictyochaeta fuegiana. Studies in Mycology 50: $171-186$.

Saccardo, P.A. 1886. Syllogue Fungorum IV. Hyphomycetae. Padua.

Sampaio, E.V.S.B. 1995. Overview of the Brazilian Caatinga. Pp. 35-58. In: S.H. Bullock, H.A. Mooney \& E. Medina (eds.). Seasonally dry forests. Cambridge, Cambridgge University Press.

Schoenlein-Crusius, I. H. \& Grandi, R. A. P. 2003. The diversity of aquatic Hyphomycetes in South America. Brazilian Journal of Microbiology 34: 183-193.

Schoenlein-Crusius, I.H. \& Milanez, A.I. 1990. Hyphomycetes aquáticos no Estado de Sao Paulo, Brasil. Revista Brasileira de Botânica 13: 61-68.

Schoenlein-Crusius, I.H.; Milanez, A.I.; Trufem, S.F.B.; Pireszottarelli, C.L.A.; Grandi, R.A.P.; Santos, M.L. \& Giustra, K.C. 2006. Microscopic fungi in the Atlantic rainforest in Cubatão, São Paulo, Brazil. Brazilian Journal of Microbiology 37: 267-275.

Shenoy, B.D.; Jeewon, R.; Wu, W.P.; Bhat, D.J. \& Hyde, K.D. 2006 Ribosomal and RPB2 DNA sequence analyses suggest that Sporidesmium and morphologically similar genera are polyphyletic. Mycological Research 110: 916-928.

Silva, P. 2007. Fungos Anamorfos decompositores do folhedo de Caesalpinia echinata Lam. provenientes de exemplares estabelecidos em áreas com e sem impacto de poluição aérea. Dissertação de mestrado. Instituto de Botânica, São Paulo.

Somrithipol, S.; Sudhom, N.; Tippawan, S. \& Jones, E.B.G. 2007. A new species of Falcocladium with turbinate vesicles from Thailand. Sydowia 59: 148-153

Stalpers, J. 2007. Filamentous fungi database. CBS - Centraalbureau voor Schimmelcultures. Disponível em: <http://www.cbs.knaw.nl/>. (Acesso: 12/10/2007).

Subramanian, C.V. 1992. A reassessment of Sporidesmium (Hyphomycetes) and some related taxa. Proceedings of the Indian National Sciences Academy 58: 179-190.

Sutton, B.C. 1973. Hyphomycetes from Manitoba and Saskatchewan, Canada. Mycological Papers 132: 1-143.

Sutton, B.C. 1975. Eucalyptus Microfungi. Satchmopsis gen. nov., and new species of Coniella, Coniothyrium and Harknessia. Nova Hedwigia 26: $1-15$.

Sutton, B.C. 1977. Some dematiaceous Hyphomycetes from Eucalyptus leaf litter. Boletin de la Sociedad Argentina de Botánica 18(1/2): 154-161.

Sutton, B.C. \& Hodges Junior, C.S. 1975a. Eucalyptus microfungi: Codinaea and Zanclospora species from Brazil. Nova Hedwigia 26: 517-525

Sutton, B.C. \& Hodges Junior, C.S. 1975b. Eucalyptus microfungi: two new Hyphomycetes genera from Brazil. Nova Hedwigia 26: 527-533.

Sutton, B.C. \& Hodges Junior, C.S. 1976a. Eucalyptus microfungi: Microdochium and Phaeoisaria species from Brazil. Nova Hedwigia 27: $215-222$.

Sutton, B.C. \& Hodges Junior, C.S. 1976b. Eucalyptus microfungi: some setose Hyphomycetes with phialides. Nova Hedwigia 27: 343-352.

Sutton, B.C. \& Hodges Junior, C.S. 1976c. Eucalyptus microfungi: Mycoleptodiscus species and Psedoutracylla gen. nov. Nova Hedwigia 27: 693-700.

Sutton, B.C. \& Hodges Junior, C.S. 1977. Eucalyptus microfungi: Miscellaneous Hyphomycetes. Nova Hedwigia 28: 487-498.

Sutton, B.C. \& Hodges Junior, C.S. 1978. Eucalyptus microfungi: Chaetendophragmiopsis gen. nov. an other Hyphomycetes. Nova Hedwigia 29: 593-607.

Sutton, B.C. \& Mel'nik, V.A. 1992. Phaeostalagmus rosicus sp. nov. from Russia. Mycological Research 96: 908-910.

Tabarelli, M. \& Silva, J.M.C. 2003. Áreas e ações prioritárias para a conservação da Caatinga. Pp. 777-795. In: I.R. Leal; M. Tabarelli \& J.M.C. Silva (eds.) Ecologia e conservação da Caatinga. Recife, Editora Universitária, Universidade Federal de Pernambuco.

Tang, A.M.C.; Jeewon, R. \& Hyde, K.D. 2005. Succession of microfungal communitites on decaying leaves of Castanopsis fissa. Canadian Journal of Microbiology 51: 967-974.

Tsui, C.K.M.; Berbee, M.L.; Jeewon, R. \& Hyde, K.D. 2006. Molecular phylogeny of Dictyosporium and allied genera inferred from ribosomal DNA. Fungal Diversity 21: 157-166. 
Wu, W. \& Zhuang, W. 2005. Sporidesmium, Endophragmiella and related genera from China. Fungal Diversity Research Series 15 . Hong Kong, Fungal Diversity Press.

Yanna; Ho, W.H. \& Hyde, K.D. 2001. Sporidesmiella oraniopsis, a new species of dematiaceous hyphomycetes from North Queensland, Australia and synopsis of the genus. Fungal Diversity 8: 183-190.

Zak, J.C. 1993. The enigma of desert ecosystems: the importance of interactions among the soil biota to fungal biodiversity. Pp. 5971. In: S. Isaac, J.C. Frankland, R. Watling \& A.J. Whalley (eds.) Aspects of Tropical Mycology. Cambridge, Cambridge University Press,

Zhou, D.Q.; Hyde, K.D. \& Wu, X.L. 2001. New records of Ellisembia, Penzigomyces, Sporidesmium and Repetophragma species on bamboo from China. Acta Botanica Yunnanica 23: 45-51. 